\title{
A.I. Based Image Segmentation
}

\author{
S.Vitulano, C.Di Ruberto, M.Nappi \\ Faculty of Medicine, University of Cagliari, ITALY \\ E-Mail vitulano@facmed.unica.it, fax (39) 70-663651
}

\begin{abstract}
The paper will show a possible model of the human perceptive process. With the aim to implement the model we have introduced a well know technique of Problem Solving.

The most important roles of our model are played by the Evaluation Function and the control strategy. The Evaluation Function is related to the ratio between the entropy of one region or zone of the picture and the entropy of the entire picture.

The model has been widely experimented on different kinds of pictures: natural scenes, CT Scan, MR and mammographies.
\end{abstract}

\section{Introduction}

Segmentation is a significant issue in the field of image processing and image understanding. The segmentation is the process, both human and automatic, that individuates in a pictorial scene zones or regions showing some characteristics with respect to a certain uniformity predicate (UP). In our case, the UP is determined by means of evaluation function $(E F)$.

It is possible to choose as $E F$ local measures as: the grey tone, the colour, the application of a local operator (gradient, laplacian, average, Sobel, etc, ) filters or global measures as Fourier transform, histogram, fractal dimension, entropy and so on. In the computer vision literature, the whole segmentation process is often shown by two opposite but related phases: Merge-and-Split.

The merge phase tries to obtain the maximum possible organisation of the picture, enhancing the transition zones between different regions.

We consider as regions all those zones of the picture whose entropy is negligible and for which it is possible to determine a statistical function or mathematical structure describing them. On the contrary, for those regions that present a high value of entropy it is not possible to determine a statistical function or a mathematical structure but, perhaps, a catastrophe. We say that the zones of the picture where entropy is high correspond to the contour or silhouette of the object.

The purpose of the split phase is to divide each region, individuated by the merge phase, in subregions which can be described by means of different mathematical structures; for these subregions the entropy changes are very small.

The rest of this paper is organised as follows: in section II we introduce a segmentation algorithm based on A.I. technique; experimental results are discussed and shown in section III, while final conclusions are given in section IV. 


\section{The Proposed Algorithm}

The task of this phase is to obtain information about the different regions of the picture: the number of existing regions, their areas and topological positions in the domain of the image, their features and their contours.

For the choice of the characteristics, of each region, and their quantification we have resorted to a typical A.I. strategy, Problem Solving, and we define:

Physical space. The domain of the picture will be the physical space of the problem and will usually be a square matrix of $512 \times 512$ pixels;

Research tree. A hierarchical structure to represent the state of the problem during its various phases is utilised, for instance the quad-tree.

Evaluation function. The histogram can give some useful information about the picture under investigation, such as entropy, grey level distribution and so on. For example, an image that is totally-homogeneous consisting of a single grey level, has a histogram with only one peak; we can associate with the peak a Gaussian distribution that has zero standard deviation, $\sigma$. Therefore, the entropy of the whole picture is zero. In other words, the entire scene is homogeneous and it is composed by only one region characterised by a single grey level, then, we can state that the entropies of both the region and the pictorial scene are zero. On the contrary, if we consider a picture with only two grey levels, white and black for instance, its histogram has two peaks. If we associate a Gaussian function with each one of these peaks, its standard deviation is different from zero. The entropy of the whole picture is different from zero and it is related to $\sigma_{1}+\sigma_{2}$, i.e. the sum of the two standard deviations. Such an image could be a regular structure (chess-board) or a chaotic scene (black and white noise). In this picture two or more homogeneous regions could exist, with entropy equal to zero, but the entropy of the whole picture is different from zero.

Usually, a real picture histogram is very complicated to read, even if it gives us some information about the whole image. This information is not enough to characterise the regions contained inside the image. It is possible to make a remark: the entropy of each region is small since it represents a function of the whole image entropy. For this reason $E F$ as entropy measure of each child domain with respect to the entropy of the whole of the domain of the father has been chosen. So the $E F$ is:

$$
E F_{c}=\frac{f_{c}\left(\sigma_{c}\right)}{f_{f}\left(\sigma_{f}\right)}
$$

where: the function $f_{c}\left(\sigma_{c}\right)$ is a measure of the entropy of the child and where $\sigma_{c}$ is the sum of the standard deviations of the peaks in the histogram of the child $c$

and $\quad f\left(\sigma_{f}\right)$ is a measure of the entropy of the father and where $\sigma_{f}$ is the sum of the standard deviations of the peaks in the histogram of the father.

The lists. In order to store the quad-tree's nodes visited during the various search phases some lists are utilised; they are: 
the F-Open list contains the nodes generated along an optimal path but not yet expanded, they are ordered in the list with respect to the $E F$ values;

the F-closed list contains the nodes expanded along an optimal path;

the $B$-closed list contains the nodes whose associated domain covers a region already individuated.

The rules. The choice of the rules plays a significant role in the field of Problem Solving; in fact, it can influence the number of operations necessary to reach the problem solution.

The control strategy. The purpose we intend to realise in this phase is to highlight, inside the pictorial scene, those zones whose entropy is zero or quasi-zero. Better, the zones individuated as regions are those ones whose $E F$ is stable or gradually decreasing. So, we can state:

if $Q$ is the domain of a region then there exists a set $H=\left\{H_{l}, H_{2}, \ldots, H_{n}\right\}$ of subdomains of $Q$ such that $H_{n} \subseteq H_{n-1} \subseteq \ldots \subseteq H_{l} \subseteq Q$ and whose $E F$ value is less than or equal to the $E F$ value of $Q$.

Such a set $H$ has a subdomain whose $E F$ value is higher than the $E F$ value of $Q$ as lower bound. We call this subdomain piece of the region.

The Backward strategy. We have said that along an optimal path the $E F$ assumes constant or decreasing values. Along such a path, when the dimensions of the subdomain relative to a level of the search tree are less than the dimensions of the domain of the piece, then a sudden variation of the $E F$ value occurs. This means that the dimensions of these nodes are smaller than the dimensions of the piece of the region. The backward strategy allows to determine exactly the dimensions of the piece.

The split phase. The aim of the splitting phase is to distinguish the structures associated with each region whatever their statistics be.

The merging phase divides the image into regions whose elements satisfy a predicate of uniformity, but the fact that inside the same region two elements satisfy its uniformity predicate does not imply that they have the same texture or structure. The structure associated with an element is a description of the mutual relation among the pixels contained in the partition element. The basic task of the split phase is to associate a structure with the region under investigation or to split the region in more regions.

\section{Experimental Results}

Let's consider, as a first example, a theoric picture where all the grey tones contained in its histogram are uniformly distributed in the picture domain. 


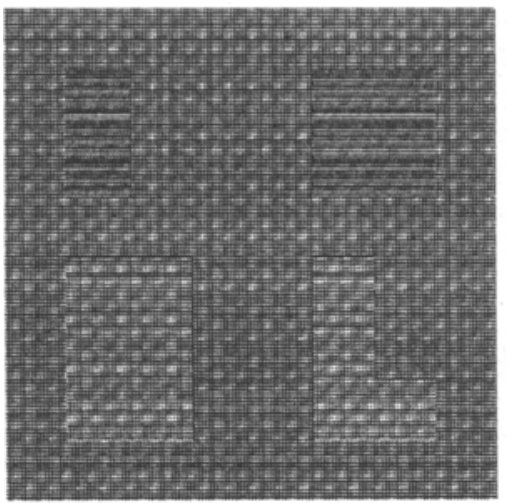

(a)

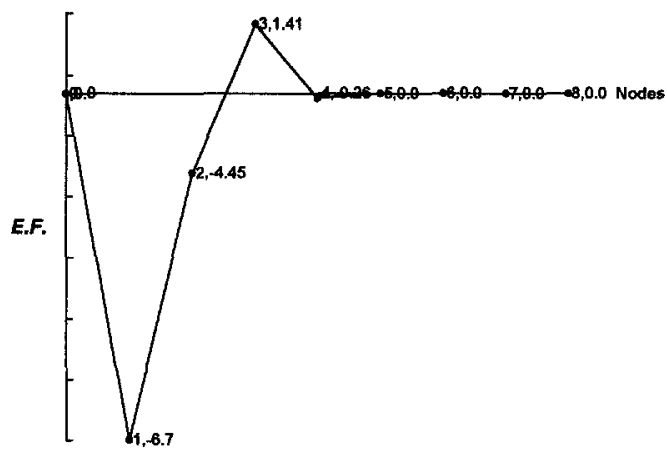

(c)

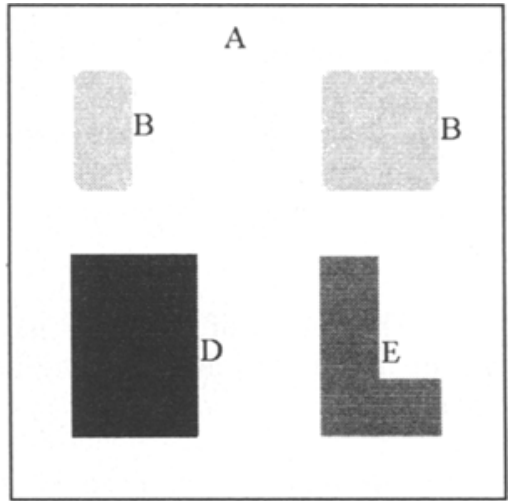

(b)

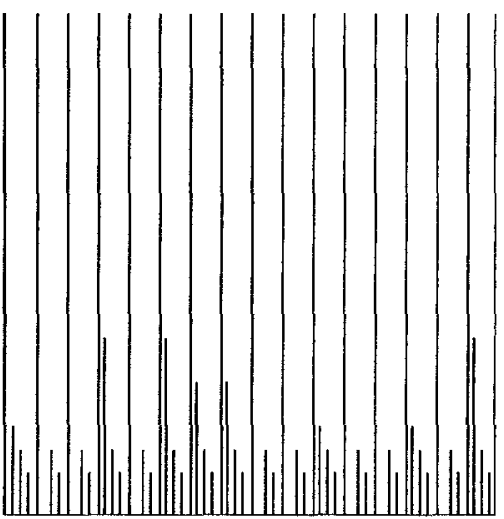

(d)

Fig. 1

In fig 1a) it is shown the input picture; in (b) it is shown the result obtained applying the segmentation algorithm proposed; in (c) is shown the behaviour of the E.F. and in (d) the histogram of the picture in (a).

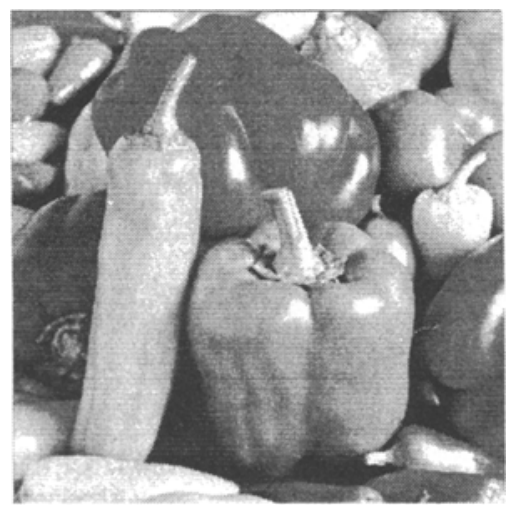

(a)

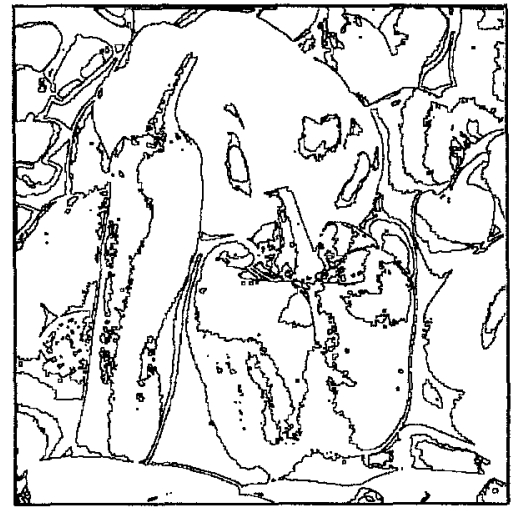

(b)

Fig.2

In (a) it's shown a well known picture: peppers and in (b) are shown the contours obtained applying our method. 
Many authors propose local operator in order to obtain the contour of the objects contained in a scene. In fig 2 it's shown the contours obtained applying our method to a well known picture.

Let us show the results obtained of our method to a real picture: a CT picture of a human brain.

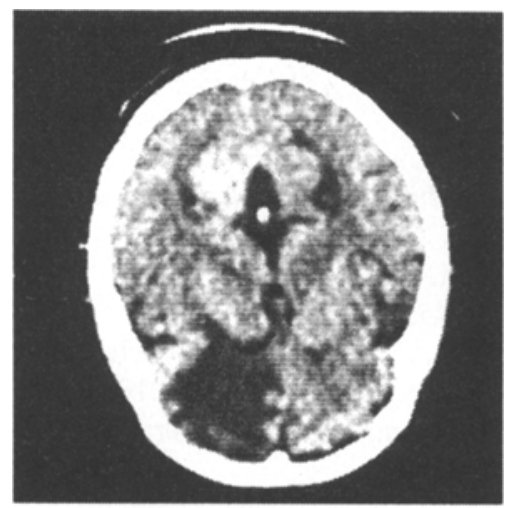

(a)

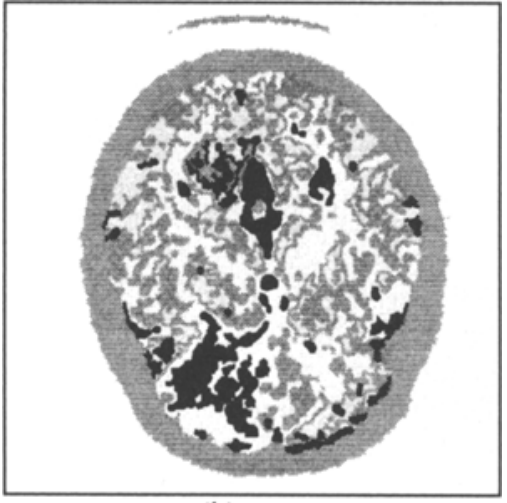

(b)

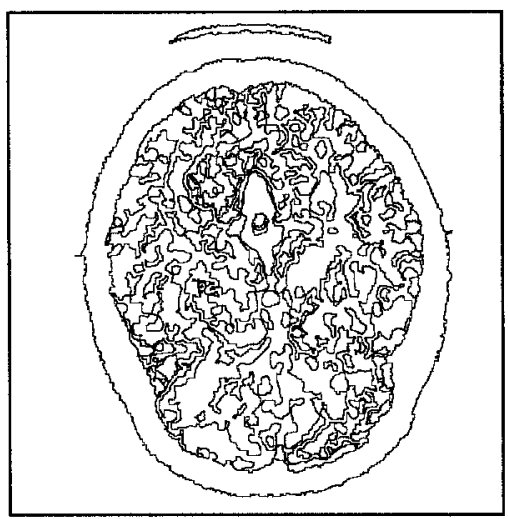

(c)

Fig.3

In (a) it is shown a real picture: CT picture of a human brain; in (b) it is shown the segmented picture and where each grey-tone represents a region; in (c) it is shown the contours between the different regions.

\section{Conclusion}

The wide experimentation of our model on different kinds of image allows us to formulate the following considerations:

the method proposed here gives information not only about the regions contained in the scene (small entropy) but also about the contours of the regions themselves. In fact contours are the transition zones between regions (high entropy). A comparison between the method here proposed and local operators strategy allows us to make the following considerations: it is not necessary to choose the size of local operators, it is 
not necessary introduce thresholds ad hoc, it is not time consuming (our algorithm is $\mathrm{O}(n \log n))$ and, finally, are not necessary preliminary iterated smoothings: smoothing procedure is absolutely forbidden for medical pictures. We wish, in the next future, to show the results obtained comparing our method with the ones proposed in (15).

We have widely experimented the method here proposed on different types of pictures, i.e. medical, natural and textures images.

\section{References}

1. R.M.Haralick, Statistical and Structural Approaches to Textures, Proc. IEEE 67, pp.786- 804, 1979

2. J.M.Keller, S.Chen, R.M. Crownover, Texture "Description through Fractal Geometry", C.V.G.I.P., v.45, pp.150-166, 1989.

3. N.Sarkar, B.B.Chaudhuri, "An Efficient Approach to Estimate Fractal Dimension of Textural Images", Patt. Recog., v.25, n.9, pp.1035-1041, 1992.

4. H.Samet, "The Quadtree and Related Hierarchical Data Structures", ACM Computers Surveys 1984.

5. R.M.Haralick, L.Shapiro, "Survey: Image Segmentation Techniques", Computer Vision Graphics and Image Processing, 29, pp. 100-132, 1985.

6. A.Rosenfeld, A.C.Kak, Digital Signal Processing, New York, 1982.

7. J.Beaulieu, M.Goldberg, "Hierarchy in Picture Segmentation: a Stepwise Optimization Approach", IEEE Trans. on PAMI, v.11, n.2, 1989.

8. A.Rosenfeld, G.J.Vanderbrug, "Coarse-Fine Template Matching", IEEE Trans. on System, Man and Cybernetic, Feb. 1977.

9. I.Prigogine, I.Stengers, La nuova alleanza. Metamorfosi della Scienza, Einaudi, 1981.

10. C.Di Ruberto, R.Prudente, S.Vitulano, "Segmentation of Biomedical Images: CT and MNR", Digital Signal Processing '91, Ed.: V.Cappellini e A.G. Constantidines, Elsevier Science Publishers, 1991.

11. C.Di Ruberto, M.Nappi, S.Vitulano, "Problem Solving in a Vision Model", Computer Application in Industry, Ed. M.H.Hamza, ACTA Press, .

12. P.Brodatz, Textures: A Photographic Album for Artists and Designers, New York: Dover, 1966.

13. P.J.Burt, T.Hong, A.Rosenfeld, "Segmentation and Estimation of Image Region Properties Through Cooperative Hierarchical Computation", IEEE Trans. on System, Man and Cybernetics, vol.11, no.12, 1981.

14. C.Di Ruberto, N.Di Ruocco, S.Vitulano "A Segmentation Algorithm based on AI Techniques", Pattern Recognition in Practice IV, ED. E.S. Gelsema and L.N. Kanal, Elsevier Science Publishers pp 153- 164, 1994.

15. W.E. Higgins, C. Hsu, "Edge Detection Using Two-Dimensional Local Structure Information", Pattern Recognition, vol. 27, n.2, pp. 277-294, 1994.

16. R.M. Haralick, "Digital step edges from zero crossing of second directional derivatives", IEEE trans. pattern Analysis Mach. Intell., Pami 6 58- 68 (1984)

17. F Tomita and S. Tsuyi, "Extraction of multiple regions by smoothing in selected neighbourhoods", IEEE Trans, Syst. Man. Cybernetics SMC-7,107 109 (1977). 